\title{
A Kinematic Thumb Model for the ACT Hand
}

\author{
Lillian Y. Chang and Yoky Matsuoka \\ The Robotics Institute, Carnegie Mellon University \\ Pittsburgh, Pennsylvania 15213 \\ Email: \{lillianc, yoky\}@cs.cmu.edu
}

\begin{abstract}
The thumb is essential to the hand's function in grasping and manipulating objects. Previous anthropomorphic robot hands have thumbs that are biologically-inspired but kinematically-simplified. In order to study the biomechanics and neuromuscular control of hand function, an anatomical robotic model of the human thumb is constructed for the anatomically-correct testbed (ACT) hand. This paper presents our ACT thumb kinematic model that unifies a number of studies from biomechanical literature. We also validate the functional consistency (i.e. the nonlinear moment arm values) between the cadaveric data and the ACT thumb. This functional consistency preserves the geometric relationship between muscle length and joint angles, which allows robotic actuators to imitate human muscle functionality.
\end{abstract}

Index Terms - Humanoid Robots, Biologically Inspired Systems, Biomechanics, Hand Surgery, Anatomically-Correct Testbed

\section{INTRODUCTION}

An anatomically-correct testbed (ACT) hand is being developed to provide the following tools for novel research:

1. As a functional physical model of a human hand for neuro- and plastic-surgeons to analyse surgical reconstruction techniques for impaired hands,

2. As an experimental testbed to study the complex neuromuscular control of the human hand, and

3. As a telemanipulator that mimics both the passive and active properties of a human hand for precision teleoperation and prosthetics.

Although researchers have developed many variations of biologically-inspired hands, there is not yet a robotic hand which can accomplish the goals listed above because none are anatomically-correct. Often anthropomorphic robotic hands have reduced degrees of freedom or joint axes and actuators that do not correspond to the human counterparts [1-4]. Educational and medical models of bones, joints, and muscles may be valuable as static anatomical references but are inadequate functional devices. In order to investigate the three objectives above, researchers are designing an ACT hand that preserves the key features and properties that define a human hand's biomechanical function and neural control.

The initial effort on the ACT hand concentrated on designing and building a complete index finger $[5,6]$. Essential anatomical elements of the working prototype include the number of bones and muscles, degrees of freedom at each joint, tendon insertion locations on the bone, and tendon topology of the extensor mechanism. In addition, the ACT index finger retains the complex geometry of the bone surfaces (crucial for maintaining tendon force vectors and moment arms), joint range of motion, and bone size, mass, and strength [5]. An actuator system was also developed to model passive and active muscle contribution to the finger dynamics [7].

Achieving full hand function or even minimal grasping capability requires a working thumb in combination with the index finger. The human thumb's opposability and strength is fundamental to the hand's interaction with and manipulation of objects [8,9]. Since clinicians commonly consider the thumb responsible for at least $50 \%$ of overall hand function [10], it is crucial to include an appropriate model of the thumb in the ACT hand. Some dextrous robot hands are capable of successful grasping motion with a mechanical thumb identical to the fingers except for placement in an opposed position. The Stanford/JPL hand has a three-degree-of-freedom finger placed opposite two other three-degree-of-freedom fingers [1]. Another example is the Utah/MIT hand, which has four digits with 4 degrees of freedom, with one finger place on a palm base opposed to the other three [2]. The Robonaut hand does incorporate a three-degree-of-freedom thumb kinematically distinct from the fingers [3, 4]. However, none of these mechanical thumbs is driven by a number of actuators equivalent to the number of thumb muscles. Thus they are unsuitable for studying biomechanical control in humans.

This paper presents the kinematic model of the robotic thumb for an ACT hand (Section II), the mechanical design of the thumb joints (Section III), and the incorporation of tendon structures into the model (Section IV). Section V presents the experimental validation and results of the moment arm measurements. We conclude in Section VI with a discussion of how the thumb prototype might be integrated with an anatomically-correct index finger to form the basis of an anatomical robot hand.

\section{KINEMATIC MODEL OF THE THUMB}

The kinematic properties of the ACT thumb should mimic those of a human thumb. Matching the robotic thumb joints to the anatomic joints creates the proper relative motion between the bones. Working from the wrist up to the thumb tip, the four bones of the thumb are the trapezium carpal bone, the first metacarpal, the proximal phalanx, and the distal phalanx. The three thumb joints are named the carpometacarpal (CMC), metacarpophalangeal (MCP), and interphalangeal (IP) joints, as shown in Fig.1

The classification of each articulating joint includes the number of degrees of freedom as well as the location of the rotational axes. The human thumb has been described by a variety of kinematic models [8, 9, 11-14]. Although several researchers describe the IP joint has having one rotational degree of freedom $[11,12,14]$, there is less consensus on the characterization of the MCP and CMC joints. Previous 


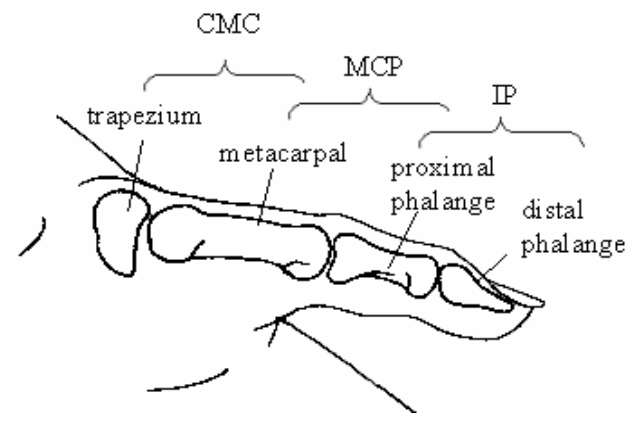

Figure 1. Bones and joints of the thumb.

research $[11,12]$ investigating thumb motion considered both the MCP and CMC joints to be universal joints, which have two perpendicular and intersecting axes.

We chose to model the thumb as having five degrees of freedom. As illustrated in Fig. 2, the IP joint has a flexionextension (FE) axis and the MCP and CMC joints each have one flexion-extension axis and one adduction-abduction (AA) axis. These are the five axes reported by [13, 14], based on a method which mechanically locates the rotational axes in cadaver hand specimens. Results of $[13,14]$ suggest that the thumb's MCP and CMC joints are not universal joints, because the FE and AA axes found in the cadaver hands were non-orthogonal and non-intersecting. Recent research [15] has used Denavit-Hartenberg robotic notation to simulate a five link model of the thumb [16] with the same kinematic description as $[13,14]$.

The ACT hand requires a thumb with these five anatomic axes instead of the simpler universal joint model to accurately represent hand biomechanics. For example, computer simulation of a thumb model with intersecting and orthogonal axes at the universal joints for the MCP and CMC predicted unrealistic forces generated at the thumbtip [17], which would be undesirable in achieving our three stated objectives for the ACT hand. In addition, modeling the robotic thumb with the non-perpendicular and offset rotational axes reported in $[13,14]$ provides the kinematic basis for other important anatomic characteristics, such as muscle moment arms about a given rotational axis. Using the more complex model of non-orthogonal and nonintersecting joints more accurately preserves the workspace of the thumb. This is achieved without added actuation complexity, because the motors are connected indirectly to the bones through the tendons instead of being mounted directly within the joint as in other robotic hands.

Other literature proposes a third axis in the $\mathrm{CMC}$ joint to account for the axial rotation of the thumb, as reviewed in $[13,14]$. Our kinematic model of the thumb does not include a third degree of freedom at the CMC joint, because [11] demonstrated that axial rotation for pronationsupination was not independent of the flexion-extension and adduction-abduction angles. The ACT thumb does not currently incorporate a load-dependent translational degree of freedom for the trapezium bone as suggested by [15], although this feature could be introduced once the thumb is integrated into an ACT robot hand design.

\section{MeChaniCAL Design of JoINTS}

Although our robotic thumb attempts to reproduce human thumb anatomy, the mechanical implementation of the joints reproduces only those features required for equivalent kinematic function. Complete duplication of the complex articular cartilage topology and synovial tissue constraints around the bones could also create the appropriate degrees of freedom. However, this would then require regenerative artificial tissue to compensate for material wear of the soft tissue as well as fluid lubrication to substitute for synovial fluid in human joints. Instead, the ACT thumb design achieves low friction with machined pin joints at each rotational axis and incorporates range of motion constraints through joint cavities in the bone, an approach consistent with a previous ACT index finger design [5].

\section{A. Location of joint axes in bones}

Though the finger bones in a previous implementation of the first ACT hand [6] consisted of cylindrical links of the same diameter, the current ACT finger replicates the anatomical bone shape due to the dependency of the muscle moment arm value on the shape of the bone surface in contact with the tendon [5]. We use three-dimensional thumb bone geometry from the same data source as the ACT finger.

We located the five joint axes of the thumb based on the kinematic model described in Section II. Although [13-15] document numerical data for relative placement of the joint axes in the thumb, [15] reports results from computer simulation output in terms of absolute length with a given statistical distribution. In order to account for the size scaling of our particular bone data, the ACT thumb implements the findings from the cadaver measurements in $[13,14]$ specified by non-dimensional proportions and angles.

The resulting locations of the axes within the thumb bones are shown in Fig. 2. The IP joint FE axis passes through the distal end of the proximal phalanx and is not perpendicular to the sagittal plane of the thumb. The FE and AA axes of the MCP joint are also non-orthogonal and nonintersecting, as explained above. The FE axis is fixed relative to the proximal phalanx while the AA axis is fixed relative to the metacarpal bone, but both intersect the distal end of the metacarpal bone. Unlike the MCP joint, the two non-orthogonal axes of the $\mathrm{CMC}$ joint are located in

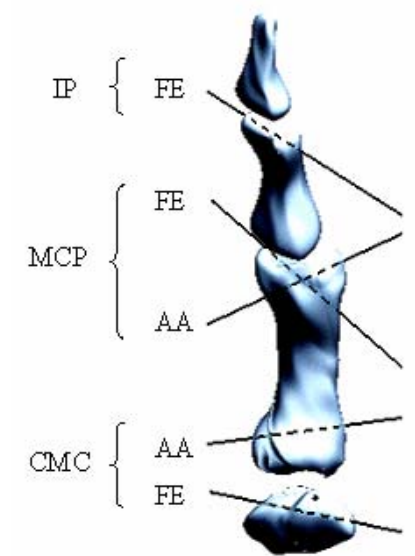

Figure 2. Kinematic model of the thumb with five rotational degrees of freedom. Joints with two degrees of freedom have non-orthogonal and non-intersecting axes. 
different bones and thus more drastically offset. The CMC AA axis passes through the proximal end of the metacarpal bone, while the CMC FE axis intersects with the trapezium carpal bone.

\section{B. Joint Range of Motion}

Although the joint angle for a revolute joint is a free kinematic variable [18], constraints in the human thumb limit the joint angle around an axis to a subset of values less than the complete 360 degrees. Biologically, ligaments and other soft tissue around the joint capsule restrict relative movement of the bones to a range of angles. In the constructed thumb, anatomically-correct ranges of motion are maintained through joint cavities cut in the solid bone.

The literature reports a variety of motion ranges for the three thumb joints based on both cadaver studies and human experiments $[11,12,19,20]$. We based our values primarily on the results of [11], which reports the active ranges of motion for different degrees of freedom based on the thumbs of a consistent subject group. We supplement this with values from [12] for the IP FE range of motion.

One source of uncertainty is the definition of the thumb's neutral position, in part due to the lack of an accepted terminology for its planes of motion. The range of motion reported by $[11,12]$ only indicates the range of flexion-extension or adduction-abduction movement but not the absolute location of the swept angle range. Knowing the neutral position, which defines where these ranges were measured from, would eliminate ambiguity of where the joint cavities should be in the ACT thumb bones. An offset joint range of motion in the model would result in incorrect muscle moment arms, which are a function of joint angle as reported by [20]. Our goal was to match the muscle moment arms with reported values for the thumb. However, the moment arm data in [20] is reported for a different range of angles based on in vitro cadaver measurement, which may not coincide with the in vivo results from [11].

The designed range of motion (Table 1) for the ACT thumb thus targets the values reported by [11] but includes additional space at the joint cavity extremes. For this first iteration, a neutral position of the thumb is approximated based on the posture of the three-dimensional bone data. Larger adjustment was made for the two adductionabduction ranges of motion due to greater uncertainty of the neutral location. The widened cavity allows moment arm measurement over a more flexible range of motion. Comparison with data reported in [20] can then determine the necessary adjustment to the neutral position for a following iteration with correct target ranges.

TABLE 1.

JOINT RANGE OF MOTION FOR ACT THUMB ROTATIONAL AXES COMPARED TO VALUES REPORTED IN LITERATURE

\begin{tabular}{|l|c|c|c|c|}
\hline $\begin{array}{c}\text { Thumb } \\
\text { axis }\end{array}$ & $\begin{array}{c}\text { Cooney } \\
{[11]}\end{array}$ & $\begin{array}{c}\text { Katarincic } \\
{[12]}\end{array}$ & $\begin{array}{c}\text { Smutz } \\
{[20]}\end{array}$ & $\begin{array}{c}\text { ACT target }+ \\
\text { widening }\end{array}$ \\
\hline IP FE & & 95 & 80 & $95+10$ \\
\hline MCP FE & $56 \pm 15$ & & 70 & $70+10$ \\
\hline MCP AA & $19 \pm 8.8$ & & 30 & $20+20$ \\
\hline CMC FE & $53 \pm 11$ & & 45 & $50+10$ \\
\hline CMC AA & $42 \pm 4$ & & 40 & $50+20$ \\
\hline
\end{tabular}

\section{Physical joint design}

The three joints of the thumb require individual mechanical designs because of the differences in the number of degrees of freedom and separation between axes.

1) IP single hinge joint: The IP joint design consists of single pin joint to represent the flexion-extension degree of freedom between the two phalangeal bones. A link arm rigidly attached to the distal phalange rotates about an axle coinciding with the IP FE axis in the proximal phalange (Fig. 3A). The spatial separation imposed by the link arm is based on the natural separation between the bones from the thumb scan source. In a human hand, cartilage and synovial tissue would fill this space to maintain proper distance between bones for smooth motion.

The geometry of the articulating bone ends was maintained except for a narrow slot that allows the small diameter link arm to rotate around the IP FE axis pin. The span of the cavity enforces the joint range of motion to the targeted value in Table 1.

2) MCP gimbal: The MCP joint required a miniature gimbal design to physically implement the two degrees of freedom for flexion-extension and adduction-abduction between the metacarpal and proximal phalange. A larger gimbal design would necessitate a substantial joint cavity to allow for motion along both rotational axes, removing a crucial area of the bone surface geometry that contacts the extensor mechanism [5].

The design of the gimbal is similar to that for the ACT index finger in [5], modified to incorporate the axes' nonorthogonality and offset. Supported by a pair of miniature ball bearings, the gimbal piece rotates around the MCP AA axis fixed within the metacarpal bone. A small pin joint in the gimbal piece represents the MCP FE axis, which is fixed relative to the proximal phalange via a link arm. A bend in the link arm allows for a reduced slot cut, mainly in the metacarpal palmar surface, to preserve as much of the threedimensional bone solid as possible (Fig. 3B). The sweep of the joint cavity restricts the movement of the gimbal assembly to the appropriate MCP joint range of motion.

3) $C M C$ double hinge joint: The $\mathrm{CMC}$ joint involves two pin joints at the ends of a single link arm to realize the flexion-extension and adduction-abduction degrees of freedom. Though the CMC and MCP joints are conceptually similar in that they both have $\mathrm{FE}$ and $\mathrm{AA}$ degrees of freedom, a gimbal design is not suitable for the CMC joint because its two rotational axes are located in separate bones.

The link arm between the carpal and metacarpal bone maintains the proper separation while allowing rotation about both axes. One pin joint coincides with the CMC AA axis in the proximal end of the metacarpal, while the other pin joint represents the $\mathrm{CMC} F E$ axis, which intersects the trapezium carpal bone (Fig. 3C). Joint range of motion for each of the two axes is constrained by narrow slot cuts in the metacarpal and trapezium bones.

\section{Construction and assembly}

Each of the four thumb bones was printed in ABS plastic using a fuse deposition modeling (FDM) machine (Stratasys FDM3000). The three-dimensional bone data modified with the designed joint cavities were downloaded from our $\mathrm{CAD}$ package directly to the surface curves files 

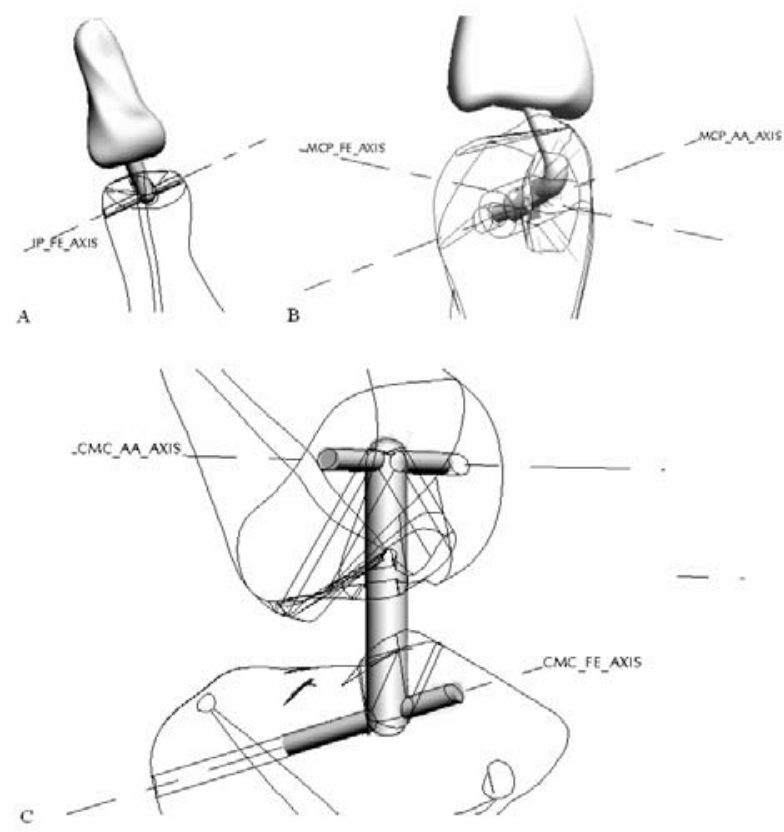

Figure 3. Individual mechanical joints.

A. Single hinge IP joint.

B. Miniature gimbal MCP joint.

C. Double hinge $\mathrm{CMC}$ joint.

required for the FDM process. Some of the small diameter holes for the hinge joint axles were re-drilled on the milling machine because the FDM support material created a ridged hole surface with inadequate diameter.

Joint link arms were manually machined from $1 / 8$ " stainless steel rod, while the MCP gimbal piece was turned and milled from 3/16" oil hardening drill rod. Aluminum $1 / 16$ " dowel pins cut to length served as hinge joint axles.

Assembly of the ACT thumb consisted of affixing link arms and gimbal bearings to the solid bone models with multi-purpose adhesive and then inserting each axle pin through the corresponding bone and link arm. A rod inserted into the proximal surface of the carpal bone connects the thumb to an aluminum plate representing the plane of the palm at the base of the thumb (Fig. 4).

\section{TENDON MODEL AND IMPLEMENTATION}

Human movement is actuated by muscles which execute neural activation signals originating from the brain.

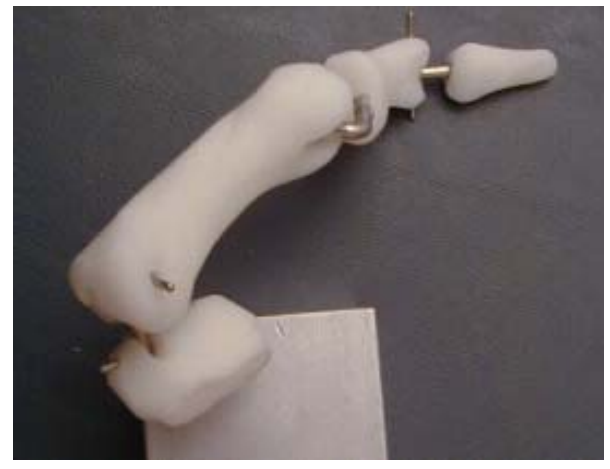

Figure 4. Assembly of bones and link arms. Note that the pins used are larger than in the final design for visibility of the joint axles.
The muscles which insert on the thumb include the flexor pollicis longus (FPL), flexor pollicis brevis (FPB), extensor pollicis longus (EPL), extensor pollicis brevis (EPB), abductor pollicis longus (APL), abductor pollicis brevis (APB), opponens pollicis (OPP), and adductor pollicis (ADP) with transverse and oblique transverse origins (ADPt, ADPo). In addition, the first dorsal interosseous (FDI) inserts onto the index finger but originates from the thumb.

Since tendons are the physical connection between bones and muscle, their orientation determines the transfer of muscle contractile force to joint rotation. The current iteration of the ACT thumb models the muscle tendon with nylon cable affixed to points along the bone surface. Insertion points were based on anatomical hand data in [21, 22]. Motion of an actuated joint depends on the force line of action as well as the point of application. In particular, it is important to preserve the tendon moment arm, which is the geometric relationship between tendon excursion and joint angle.

Tendons that control the thumb act on the bones at various orientations, and their routing is primarily determined by the combination of insertion and origin points. We do not incorporate an extensor mechanism structure for the extensor muscles of the thumb. Guide holes in the mounting plate of the ACT thumb (Fig. 5) route the tendons to the appropriate origin points, mimicking the muscles' lines of action. Additional routing constraints mimic the anatomical pulleys which prevent the tendons from bowstringing in extreme positions.

\section{EXPERIMENTAL VALIDATION}

The moment arm relationship for the muscles is critical to the biomechanical mapping between the muscle actuators and the kinematic configuration. The design decisions for using three-dimensional bone geometry and adding tendon routing structure were specifically aimed at preserving the appropriate moment arm relationship at all the joint degrees of freedom. In particular, the moment arm is not constant with joint angle, as documented for the thumb in [20].

We computed the independent moment arm for each muscle over the multiple degrees of freedom. Four rotational axes were fixed in a neutral position. Each muscle actuating across the free rotational axis was

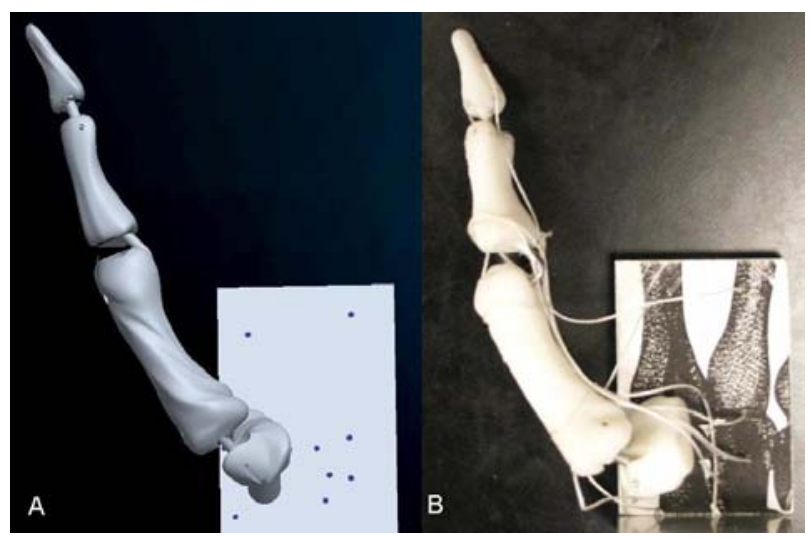

Figure 5. A. 3D computer model of ACT thumb, including insertion points. B. Assembled prototype with synthetic tendons attached at anatomical insertion points. 
independently tested by recording the joint angle and corresponding tendon excursion length. Analytically, the moment arm is the derivative of the tendon excursion with respect to joint angle [23]. We approximate the derivative from a backward finite difference:

$\frac{\partial l}{\partial \theta} \approx \frac{\Delta l}{\Delta \theta}=\frac{l(\theta)-l(\theta-\Delta \theta)}{\Delta \theta}$

where $\Delta l$ is the tendon excursion length and $\Delta \theta$ is the change in joint angle.

Out of the total 33 moment arms measured for the ACT thumb, 18 are within one standard deviation (SD) of cadaveric measurements [20] for more than half the range of motion, with 13 moment arms within 1 SD for three-fourths of the entire range of motion, and 9 moment arms within 1 $\mathrm{SD}$ for the entire range of motion. Fig. 6A shows a sample result for the ADPo tendon for the CMC AA degree of freedom, where the moment arm was within $1 \mathrm{SD}$ for the entire range of motion. Figs. $6 \mathrm{~B}$ and $6 \mathrm{C}$ show examples where the ACT thumb result was within 1 SD for threefourths or one-half of the range of motion, respectively. Although the moment arm values are not strictly within the standard deviation of cadaveric measurement, the trends of the nonlinear relationship were similar (Fig. 6B).

For the remaining fifteen moment arms, 2 are within 2 SD, 7 are within $3 \mathrm{SD}$, and 6 are within $5.5 \mathrm{SD}$ of average cadaveric thumb measurements. Figs. $6 \mathrm{D}$ and $6 \mathrm{E}$ show some sample plots of such results where the robotic thumb moment arms were not within $1 \mathrm{SD}$ for most of the range of motion, but within $2 \mathrm{SD}$ and $5.5 \mathrm{SD}$, respectively. The fact that some of the robotic thumb moment arms are outside a few SD of the mean measurement arises from the small standard deviation reported for cadaver thumbs in [20], which studied 7 hands from 6 cadaver subjects. For example, the results for the FPB tendon over the MP FE degree of freedom (Fig. 6E) is within 3.5 SD for the entire range of motion except for the data point at 50 degrees joint angle. However, even the worst cases within several SD of cadaveric measurements still match the general nonlinear trend of cadaveric measurement, where moment arms may increase or decrease with joint angle for specific portions of the range of motion.

In general, mismatches could be due to the interdependence of the moment arms, the topology of networked tendons, and the scale of the robotic thumb bones. Because several muscles span more than one joint, adjusting the insertion point may favourably change the moment arm for one degree of freedom but have the opposite effect on another degree of freedom that the same muscle actuates. Interdependency between muscle moment arms could also arise from anatomic structures such as the thumb extensor hood, which was not modelled in this prototype of the ACT thumb. In addition, the scale of the robotic thumb bones for the ACT hand may be scaled larger or smaller than the average hand studied in [20], resulting in our results being biased relative to the cadaveric measurements.

The results show that preserving rotational axes, bone surface geometry, and tendon insertion points maintains the important moment arm relationships for the independent degrees of freedom.
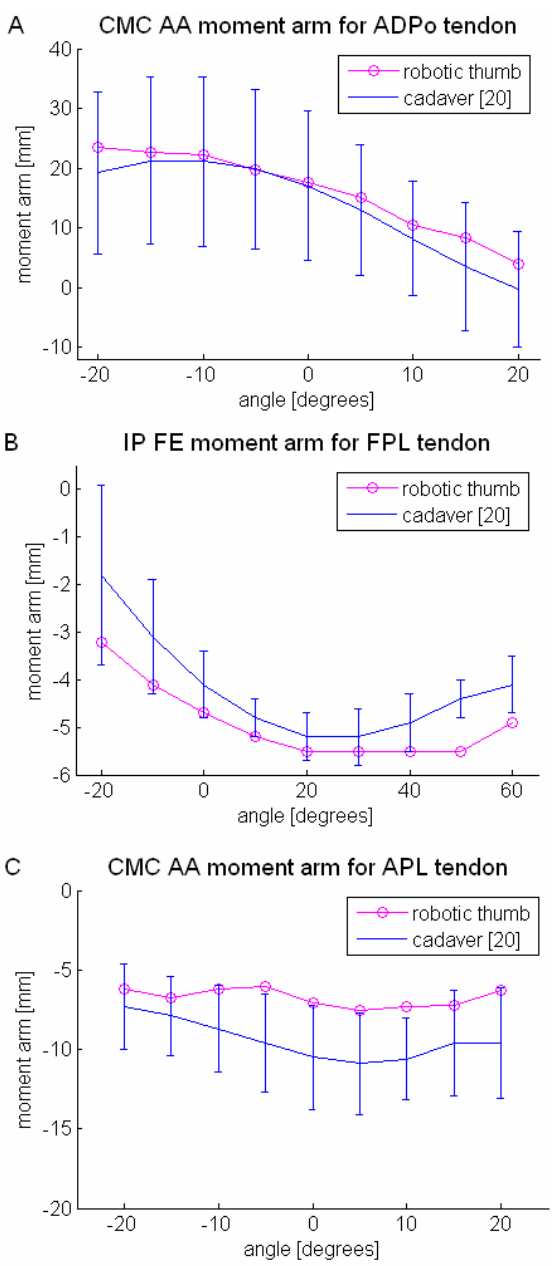

D MP AA moment arm for FPL tendon

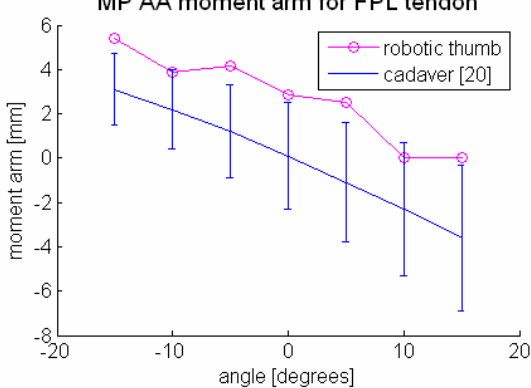

E MP FE moment arm for FPB tendon

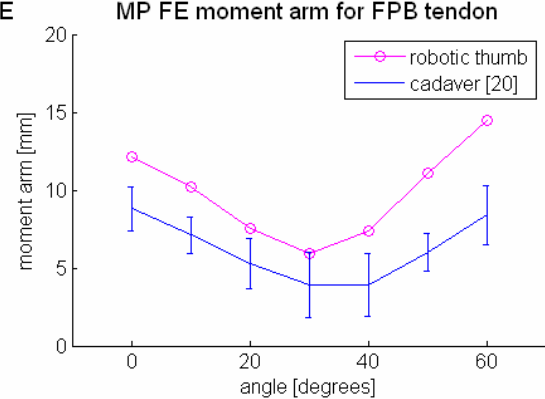

Figure 6. Moment arm measurements for the ACT thumb compared to published cadaver moment arm data reported in [20].

A. ADPo muscle for the CMC AA. B. FPL muscle for the IP FE.

C. APL muscle for the CMC AA. D. FPL muscle for the MP AA. E. FPB muscle for the MP FE. 


\section{DISCUSSION}

Our investigation highlights important features for developing an anatomical and functional model of the thumb, a crucial component of any hand activity involving manipulation and interaction with objects.

The kinematics of the ACT thumb include five degrees of freedom for the three thumb joints. We chose to design the thumb with rotational axes which were not aligned with anatomical planes of the body. In addition, axes are nonorthogonal and non-intersecting when two degrees of freedom exist at a single joint. Although implementing a simplified model with universal joints would reduce manufacturing difficulty, maintaining the complex kinematic description preserves the correct anatomic motion necessary for the ACT hand's intended research contributions.

Machined joints allow for low-friction joints without reproducing biological soft tissue constraints or using synovial-like fluid lubrication. Joint cavities impose the approximate range of motion at each degree of freedom, and their size can be modified in the future to match the neutral position of the thumb based on moment arm measurements. The one exception is joint motion of the MCP gimbal, which freely abducts and adducts at zero flexion-extension orientation but otherwise is properly constrained.

The ACT thumb currently models the tendons as individual actuators. This was sufficient for preserving several of the independent moment arm relationships. To further improve the independent moment arms of the robotic thumb, a physical model of the extensor mechanism topology may be required, as suggested by previous work on the index finger [6]. For composite joint motion, it may also be necessary to investigate models of the thumb's extensor mechanism. Future work on the ACT thumb can also extend the current prototype in several other aspects. Modified designs of the bones with adjusted joint cavities and material removal can be fabricated from aluminum to mimic biologic bone structural strength and mass. In addition, connecting the artificial tendon cables to mechanical actuators requires matching muscle active and passive properties. Ultimately, we intend to design a consolidated ACT hand that integrates the robotic thumb with a robotic index finger to form the basis of a functional hand for studying and understanding neural control of human dextrous manipulation.

\section{ACKNOWLEDGMENT}

The authors would like to thank Michael Vande Weghe for his advice on machining and fabrication of the ACT thumb. In addition, Stuart Weiler measured and calculated the moment arms. This project is supported by NSF CISE 0423546 and NIH 1 R21 EB005967-01.

\section{REFERENCES}

[1] M. T. Mason and J. K. Salibury, Robot hands and the mechanics of manipulation. Cambridge, MA: The MIT Press, 1985.

[2] I. D. McCammon and S. C. Jacobsen, "Tactile sensing and control for the Utah/MIT hand," in Dextrous Robot Hands, T. Iberall, Ed. New York: Springer-Verlag, 1990, pp. 239-266.

[3] C. S. Lovchik and M. A. Diftler, "The Robonaut Hand: a dexterous robot hand for space," in IEEE International Conference on Robotics and Automation, 1999, vol. 2, pp. 907-912.
[4] T. B. Martin, R. O. Ambrose, M. A. Diftler, R. Platt, Jr., and M. J. Butzer, "Tactile gloves for autonomous grasping with the NASA/DARPA Robonaut," in IEEE International Conference on Robotics and Automation, 2004, vol. 2, pp. 1713-1718.

[5] M. Vande Weghe, M. Rogers, M. Weissert, and Y. Matsuoka, "The ACT hand: design of the skeletal structure," in IEEE International Conference on Robotics and Automation, 2004, vol. 4, pp. 33753379.

[6] D. D. Wilkinson, M. V. Weghe, and Y. Matsuoka, "An extensor mechanism for an anatomical robotic hand," in IEEE International Conference on Robotics and Automation, 2003, vol. 1, pp. 238-243.

[7] N. Gialias and Y. Matsuoka, "Muscle actuator design for the ACT hand," in IEEE International Conference on Robotics and Automation, 2004, vol. 4, pp. 3380-3385.

[8] I. A. Kapandji, The Physiology of the Joints, Upper Limb, 2nd ed. London: E and S Livingstone, 1970, vol. 1, pp. 182-201.

[9] F. J. Bejjani and J. M. F. Landsmeer, "Biomechanics of the Hand," in Basic Biomechanics of the Musculoskeletal System, M. Nordin and V. H. Frankel, Eds., 2nd ed. Philadelphia: Lea \& Febiger, 1989, pp. 275289.

[10] J. C. Colditz, "Anatomic considerations for splinting the thumb," in Rehabilitation of the hand: surgery and therapy, M. E. J. Hunter J. M., Callahan A. D., Ed. Philadelphia: C. V. Mosby Company, 1990.

[11] W. P. Cooney, 3rd, M. J. Lucca, E. Y. Chao, and R. L. Linscheid, "The kinesiology of the thumb trapeziometacarpal joint," J Bone Joint Surg Am, 1981, vol. 63, pp. 1371-81.

[12] J. A. Katarincic, "Thumb kinematics and their relevance to function," Hand Clin, 2001, vol. 17, pp. 169-74.

[13] A. Hollister, W. L. Buford, L. M. Myers, D. J. Giurintano, and A. Novick, "The axes of rotation of the thumb carpometacarpal joint," $J$ Orthop Res, 1992, vol. 10, pp. 454-60.

[14] A. Hollister, D. J. Giurintano, W. L. Buford, L. M. Myers, and A. Novick, "The axes of rotation of the thumb interphalangeal and metacarpophalangeal joints," Clin Orthop, 1995, vol. 320, pp. 188-93.

[15] V. J. Santos and F. J. Valero-Cuevas, "Anatomical Variability Naturally Leads to Multimodal Distributions of Denavit-Hartenberg Parameters for the Human Thumb," in Engineering in Medicine and Biology Society, 2003.

[16] D. J. Giurintano, A. M. Hollister, W. L. Buford, D. E. Thompson, and L. M. Myers, "A virtual five-link model of the thumb," Med Eng Phys, 1995, vol. 17, pp. 297-303.

[17] F. J. Valero-Cuevas, M. E. Johanson, and J. D. Towles, "Towards a realistic biomechanical model of the thumb: the choice of kinematic description may be more critical than the solution method or the variability/uncertainty of musculoskeletal parameters," J Biomech, 2003, vol. 36, pp. 1019-30.

[18] J. J. Craig, Introduction to Robotics: Mechanics and Control, 2nd ed. Reading, MA: Addison-Wesley Publishing Company, Inc., 1989.

[19] J. H. Coert, H. G. van Dijke, S. E. Hovius, C. J. Snijders, and M. F. Meek, "Quantifying thumb rotation during circumduction utilizing a video technique," J Orthop Res, 2003, vol. 21, pp. 1151-5.

[20] W. P. Smutz, A. Kongsayreepong, R. E. Hughes, G. Niebur, W. P. Cooney, and K. N. An, "Mechanical advantage of the thumb muscles," J Biomech, 1998, vol. 31, pp. 565-70.

[21] A. M. R. Agur and A. F. Dalley, Grant's Atlas of Anatomy, 11th ed. Philadelphia: Lippincott Williams \& Wilkins, 2005.

[22] B. Calais-Germain, Anatomy of Movement. Seattle: Eastland Press, 1993.

[23] K. N. An, K. Takahashi, T. P. Harrigan, and E. Y. Chao, "Determination of Muscle Orientations and Moment Arms," $J$ Biomech Eng, 1984, vol. 106, pp. 280-282. 